\title{
Empirical likelihood ratio confidence intervals for conditional survival probabilities with right censored data
}

\author{
JIAN-JIAN REN*,† AND TONYA RIDDLESWORTH
}

In the analysis of survival data, we often encounter situations where the response variable (the survival time) $T$ is subject to right censoring, but the covariates $Z$ are completely observable and are often discrete or categorical. In this article, we construct the empirical likelihood ratio confidence region for conditional survival probabilities based on bivariate data which are subject to right censoring in one coordinate and have a discrete covariate $Z$. We show that such an empirical likelihood ratio confidence region is indeed an interval, and we establish some related properties of the empirical likelihood ratio. The generalization of our results in this article to the multivariate covariate $Z$ with dimension $p>1$ is straightforward.

KEYwords AND PHRAsEs: Empirical likelihood, Maximum likelihood estimator, Right censored data.

\section{INTRODUCTION}

In the analysis of survival data, we often encounter situations where the response variable is the survival time $T$ and is subject to right censoring, but the $p$-dimensional vector $Z$ of covariates with components such as treatments, gender, etc., are completely observable. In the nonparametric setting, we are interested in an interval estimate for conditional survival probability $P\left\{T>t_{0} \mid Z=z_{0}\right\}$, where $t_{0}$ and $z_{0}$ are given values of interest. Such a problem is equivalent to constructing confidence intervals for the following conditional probability:

$$
\theta_{0}=P\left\{T \leq t_{0} \mid Z=z_{0}\right\}
$$

For simplicity of presentation, here we consider the case that covariate $Z$ is a scalar rather than a vector, i.e., $Z$ with dimension $p=1$. The generalization of our results in this article to the multivariate case with $p>1$ is straightforward.

Specifically, suppose that

$$
\left(T_{1}, Z_{1}\right),\left(T_{2}, Z_{2}\right), \ldots,\left(T_{n}, Z_{n}\right)
$$

* Corresponding author.

†The author's research was partially supported by NSF grants DMS0604488 and DMS-0905772. is a random sample of $(T, Z)$, but the actually observed survival data are the bivariate data with one coordinate subject to random right censoring as follows:

$$
\left(V_{1}, \delta_{1}, Z_{1}\right),\left(V_{2}, \delta_{2}, Z_{2}\right), \ldots,\left(V_{n}, \delta_{n}, Z_{n}\right)
$$

where $V_{i}=\min \left\{T_{i}, C_{i}\right\}, \delta_{i}=I\left\{T_{i} \leq C_{i}\right\}$, and $C_{i}$ is the right censoring variable with distribution function (d.f.) $F_{C}$ and is independent of $\left(T_{i}, Z_{i}\right)$. Note that in practical situations the covariate variable $Z$ in (1.1)-(1.3) is often discrete or categorical. In this article, we construct empirical likelihood ratio confidence intervals (ELRCI) for conditional probability $\theta_{0}$ in (1.1) based on right censored survival data (1.3), where covariate $Z$ is discrete.

The empirical likelihood approach (Owen, 1988) is a nonparametric likelihood method, thus it is an appealing procedure with broad applications in survival data analysis. We refer to Owen (2001) and a nice survey paper by Li, Li and Zhou (2005) for results on this topic. Among existing works in the literature, the one most closely related to ours is that by Li and van Keilegom (2002), where they constructed confidence intervals and bands for the conditional survival probabilities using the empirical likelihood approach. However, the problem considered by Li and van Keilegom (2002) was for a continuous covariate $Z$, and their procedure involves bandwidth selection and kernel selection. In comparison, the problem we consider in this article is of special importance in practice, and our procedure does not involve any bandwidth or kernel selection.

The rest of this article is organized as follows. In Section 2, we construct the empirical likelihood based confidence region for $\theta_{0}$ in (1.1) using the empirical likelihood based bivariate nonparametric maximum likelihood estimator (BNPMLE) $\hat{F}_{n}(t, z)$ for bivariate distribution function (d.f.) $F_{0}(t, z)$ of $(T, Z)$ with right censored survival data (1.3), which was obtained by Ren and Riddlesworth (2012). We show that such a confidence region is indeed an interval. The proofs are given in Section 3.

It should be noted that the results similar to our main theorems in Section 2 are known for empirical likelihood inference in the univariate data case, however they are not obvious and quite difficult for the case with censored bivariate data which we consider in this paper. Moreover, the computation of ELRCI and the proof of related Wilk's theorem 
are very difficult problems technically, and further careful and much more involved work is needed.

\section{EMPIRICAL LIKELIHOOD RATIO CONFIDENCE INTERVALS}

To derive the empirical likelihood function for bivariate d.f. $F_{0}(t, z)$ of $(T, Z)$ based on survival data (1.3), we let all possible values of discrete covariate variable $Z$ be given by:

$$
z_{1}, z_{2}, \ldots
$$

and let

$U_{1}<\cdots<U_{m}$ be all distinct values among $V_{1}, \ldots, V_{n}$; $Y_{1}<\cdots<Y_{q} \quad$ be all distinct values among $Z_{1}, \ldots, Z_{n}$.

Denoting

$$
\begin{aligned}
n_{i j} & =\sum_{k=1}^{n} I\left\{V_{k}=U_{i}, Z_{k}=Y_{j}\right\}, \\
\delta_{i j} & =\sum_{k=1}^{n} I\left\{V_{k}=U_{i}, \delta_{k}=1, Z_{k}=Y_{j}\right\}
\end{aligned}
$$

for $1 \leq i \leq m, 1 \leq j \leq q$, Ren and Riddlesworth (2012) show that the likelihood function for bivariate distribution function $F_{0}(t, z)$ of $(T, Z)$ with data (1.3) is given by

$$
\begin{aligned}
L(F)= & \prod_{i=1}^{m} \prod_{j=1}^{q}\left(d F\left(U_{i}, Y_{j}\right)\right)^{\delta_{i j}} \\
& \times\left(F\left(\infty, d z_{j}\right)-F\left(U_{i}, d Y_{j}\right)\right)^{n_{i j}-\delta_{i j}},
\end{aligned}
$$

where $F$ is any bivariate d.f., and denoting $P_{F}$ as the probability under $F$ we have:

$$
\left\{\begin{array}{l}
d F(t, z)=P_{F}\{T=t, Z=z\} \\
F(t, d z)=P_{F}\{T \leq t, Z=z\}=F(t, z)-F(t, z-) .
\end{array}\right.
$$

In order to derive the ELRCI for $\theta_{0}$ in (1.1), we first describe the BNPMLE $\hat{F}_{n}(t, z)$ for $F_{0}(t, z)$ by Ren and Riddlesworth (2012) as follows.

Note that (2.3) implies $n_{1 j}+\cdots+n_{m j} \geq 1$ for any $1 \leq$ $j \leq q$ and that $n_{i j}=0$ implies $\delta_{i j}=0$. Thus, letting

$$
m_{j}=\max \left\{k \mid n_{k j}>0\right\}, \quad 1 \leq j \leq q
$$

we have $n_{i j}=\delta_{i j}=0$ for all $1 \leq j \leq q, m_{j}<i \leq m$; which means that points $\left(U_{i}, Y_{j}\right)$ for $m_{j}<i \leq m$ are not observed among $\left(V_{k}, Z_{k}\right)$ 's in data (1.3), in turn, by the usual empirical likelihood treatment these points $\left(U_{i}, Y_{j}\right)$ are not assigned any probability masses. Hence, to find the BNPMLE for $F_{0}$ with likelihood function (2.4), Ren and
Riddlesworth (2012) restrict all possible candidates to those bivariate d.f.'s that assign all their probability masses to points $\left(U_{i}, Y_{j}\right)$ for $1 \leq j \leq m, 1 \leq i \leq m_{j}$ and line segments $L_{j}=\left\{\left(t, Y_{j}\right) \in \mathbb{R}^{2} ; t>U_{m}\right\}$ for $1 \leq j \leq q$, which writes likelihood function (2.4) as follows:

$$
L(F)=\prod_{j=1}^{q} \prod_{i=1}^{m}\left(p_{i j}\right)^{\delta_{i j}}\left(\sum_{k=i+1}^{m+1} p_{k j}\right)^{n_{i j}-\delta_{i j}} \equiv L(\boldsymbol{p}),
$$

where

$$
F(t, z)=\sum_{i=1}^{m} \sum_{j=1}^{q} p_{i j} I\left\{U_{i} \leq t, Y_{j} \leq z\right\}, \quad \text { for } t \leq U_{m}, z \in \mathbb{R}
$$

satisfies

$$
\left\{\begin{array}{l}
p_{i j}=d F\left(U_{i}, Y_{j}\right)=P_{F}\left\{T=U_{i}, Z=Y_{j}\right\} \\
\quad \text { for } 1 \leq j \leq q, 1 \leq i \leq m \\
p_{i j}=0, \quad \text { for } 1 \leq j \leq q, m_{j}<i \leq m \\
p_{m+1, j}=P_{F}\left\{(T, Z) \in L_{j}\right\}=P_{F}\left\{T>U_{m}, Z=Y_{j}\right\} \\
\quad \text { for } 1 \leq j \leq q \\
\sum_{j=1}^{q} \sum_{i=1}^{m+1} p_{i j}=1 .
\end{array}\right.
$$

Hence, the BNPMLE $\hat{F}_{n}(t, z)$ for $F_{0}(t, z)$ is the solution that maximizes above likelihood function $L(F)=L(\boldsymbol{p})$ in (2.7).

Let $\hat{\boldsymbol{p}}$ denote the solution of the following optimization problem:

$$
\left\{\begin{array}{l}
\max L(\boldsymbol{p})=\prod_{j=1}^{q} \prod_{i=1}^{m}\left(p_{i j}\right)^{\delta_{i j}}\left(\sum_{k=i+1}^{m+1} p_{k j}\right)^{n_{i j}-\delta_{i j}} \\
\text { subject to: } \quad \text { Constraints on } \boldsymbol{p} \text { in }(2.9) .
\end{array}\right.
$$

Ren and Riddlesworth (2012) show that in the sense of the empirical likelihood method the BNPMLE $\hat{F}_{n}(t, z)$ for $F_{0}(t, z)$ is uniquely given as stated in the following theorem.

Theorem 1. For any $1 \leq i \leq m, 1 \leq j \leq q$, we denote

$$
N_{i j}=n_{i j}+\cdots+n_{m j}=\sum_{k=1}^{n} I\left\{V_{k} \geq U_{i}, Z_{k}=Y_{j}\right\} .
$$

Then, the solution $\hat{\boldsymbol{p}}$ of (2.10) is unique and satisfies the following:

(i) For any $1 \leq j \leq q, 1 \leq i \leq m_{j}$, we have $\hat{p}_{i j}>0$ if and only if $\delta_{i j}>0$;

(ii) For any $1 \leq j \leq q, 1 \leq i \leq m_{j}$, we have $\sum_{k=i}^{m+1} \hat{p}_{k j}>0$; 
(iii) With notation $\prod_{k=1}^{0} c_{k} \equiv 1$, the BNPMLE $\hat{F}_{n}(t, z)$ is given by

$$
\left\{\begin{array}{l}
\hat{F}_{n}(t, z)=\sum_{i=1}^{m} \sum_{j=1}^{q} \hat{p}_{i j} I\left\{U_{i} \leq t, Y_{j} \leq z\right\}, \\
\quad \text { for } t \leq U_{m}, z \in \mathbb{R} \\
\hat{p}_{i j}=\left(\frac{\delta_{i j}}{N_{i j}}\right)\left(\frac{N_{1 j}}{n}\right) \prod_{k=1}^{i-1}\left(1-\frac{\delta_{k j}}{N_{k j}}\right), \\
\quad \text { for } 1 \leq i \leq m, 1 \leq j \leq q \\
\hat{p}_{m+1, j}=P_{\hat{F}_{n}}\left\{T>U_{m}, Z=Y_{j}\right\}=\left(\frac{N_{1 j}}{n}\right)-\sum_{i=1}^{m} \hat{p}_{i j}, \\
\quad \text { for } 1 \leq j \leq q
\end{array}\right.
$$

where $0 / 0$ is set as 0 whenever it occurs.

One should note that (2.3), (2.6) and (2.11) imply that for any $1 \leq j \leq q$,

$$
\left\{\begin{array}{l}
n_{m_{j}, j}>0 \Rightarrow N_{1 j} \geq N_{2 j} \geq \cdots \geq N_{m_{j}, j}>0 \\
n_{i j}=\delta_{i j}=N_{i j}=0, \quad \text { for } m_{j}<i \leq m \text { when } m_{j}<m
\end{array}\right.
$$

Thus, constraint on $\boldsymbol{p}$ in the second line of (2.9) is satisfied in (2.12).

To construct ELRCI for $\theta_{0}$ in (1.1), we define the following statistical functional:

$$
\tau(F)=P_{F}\left\{T \leq t_{0} \mid Z=z_{0}\right\}
$$

where $F$ is given by (2.8)-(2.9). Since covariate variable $Z$ is discrete and $z_{0}$ is a value of interest for $Z$, then $z_{0}$ must be one of the values in $(2.1)$, and from $0<F_{Z}\left(z_{0}\right)<1$ and Theorem 4.2.1 of Chung (1974) there exists integer $\zeta$ in (2.2) such that

$Y_{\zeta}=z_{0}$ for $1 \leq \zeta \leq q$, almost surely except finitely often.

Similarly, under assumption of $0<F_{V}\left(t_{0}\right)<1$ for d.f. $F_{V}$ of $V$ in (1.3), from Theorem 4.2.1 of Chung (1974) we have that in (2.2) almost surely except finitely often,

$$
t_{0}<U_{m}
$$

Thus, from (2.8)-(2.9) and (2.15)-(2.16) we can write (2.14) as

$$
\begin{aligned}
\tau(F) & =\frac{P_{F}\left\{T \leq t_{0}, Z=z_{0}=Y_{\zeta}\right\}}{P_{F}\left\{T \leq U_{m}, Z=Y_{\zeta}\right\}+P_{F}\left\{T>U_{m}, Z=Y_{\zeta}\right\}} \\
= & \frac{\sum_{i=1}^{m} p_{i \zeta} I\left\{U_{i} \leq t_{0}\right\}}{\sum_{i=1}^{m+1} p_{i \zeta}} \equiv \tau(\boldsymbol{p}) .
\end{aligned}
$$

For likelihood function (2.7) and the BNPMLE $\hat{F}_{n}$ given by (2.12), we know that the empirical likelihood ratio is given by $R(F)=L(F) / L\left(\hat{F}_{n}\right)$ and we denote

$$
\begin{aligned}
r(\theta) & =\sup _{F}\{R(F) \mid \tau(F)=\theta\} \\
& =\left(L\left(\hat{F}_{n}\right)\right)^{-1} \sup _{\boldsymbol{p}}\{L(\boldsymbol{p}) \mid \tau(\boldsymbol{p})=\theta\} .
\end{aligned}
$$

Then, for constant $0<c<1$ the empirical likelihood ratio confidence set $S_{n}$ for conditional probability $\theta_{0}$ in (1.1) is given by

$$
S_{n}=\{\tau(F) \mid R(F) \geq c\}=\left\{\tau(\boldsymbol{p}) \mid \boldsymbol{p} \in \mathcal{E}_{n}\right\}
$$

where $\mathcal{E}_{n}=\left\{\boldsymbol{p} \mid \boldsymbol{p}\right.$ satisfies (2.8)-(2.9) and $\left.L(\boldsymbol{p}) \geq c L\left(\hat{F}_{n}\right)\right\}$. With the proofs deferred to Section 3 , we have the following theorems on above confidence set $S_{n}$.

Theorem 2. Confidence set $S_{n}$ in (2.19) is an interval satisfying $S_{n}=\left[T_{L}, T_{U}\right]$ with

$$
T_{L}=\min _{\boldsymbol{p} \in \mathcal{E}_{n}} \tau(\boldsymbol{p}) \quad \text { and } \quad T_{U}=\max _{\boldsymbol{p} \in \mathcal{E}_{n}} \tau(\boldsymbol{p}) .
$$

Theorem 3. For $r(\theta)$ and $S_{n}$ given by (2.18) and (2.19), respectively, we have

$$
\theta_{0} \in S_{n} \quad \text { if and only if } r\left(\theta_{0}\right) \geq c \text {. }
$$

Remark 1. The results similar to above Theorem 2 and Theorem 3 are known for empirical likelihood inference in the univariate data case, however they are not obvious for the case with censored bivariate data which we consider in this current paper. In fact, the proofs are quite involved technically as shown in Section 3 as well as in Ren and Riddlesworth (2012). The computation of ELRCI $\left[T_{L}, T_{U}\right]$ given in (2.20) is also a quite difficult problem; see Riddlesworth (2011) for discussions and partial solutions on this topic. For the empirical likelihood ratio $r\left(\theta_{0}\right)$ given by $(2.18)$, we expect Wilk's theorem to hold, but the proof turns out to be very difficult in this case. For such a proof, further careful and much more involved technical work is needed, while results (2.21) in Theorem 3 is useful in this context and can be used to set constant $c$ in practice.

\section{PROOFS}

Proof of Theorem 2. Note that set $\mathcal{E}_{n}$ in (2.19) is compact, because $L(\boldsymbol{p})$ in $(2.7)$ is continuous in $\boldsymbol{p}$. Also, note that (2.6) and $L(\boldsymbol{p}) \geq c L\left(\hat{F}_{n}\right)>0$ imply the following in the product of $L(\boldsymbol{p})$ given by $(2.7)$ :

$$
0<\left(p_{m_{j}, j}\right)^{\delta_{m_{j}, j}}\left(\sum_{k=m_{j}+1}^{m+1} p_{k j}\right)^{n_{m_{j}, j}-\delta_{m_{j}, j}},
$$

in turn, from $n_{m_{j}, j}>0$ in (2.13), we have $\sum_{k=m_{j}+1}^{m+1} p_{k j}>$ 0 when $\delta_{m_{j}, j}=0 ; p_{m_{j}, j}>0$ when $\delta_{m_{j}, j}>0$; which give 
$\sum_{k=m_{j}}^{m+1} p_{k j}>0$ for any $1 \leq j \leq q$. Thus, we have

$$
\boldsymbol{p} \in \mathcal{E}_{n} \Rightarrow \sum_{k=i}^{m+1} p_{k j}>0 \Rightarrow \sum_{k=1}^{m+1} p_{k j}>0
$$

for any $1 \leq j \leq q, 1 \leq i \leq m_{j}$. Hence, $\tau(\boldsymbol{p})$ in $(2.17)$ is well-defined and continuous on compact set $\mathcal{E}_{n}$ given in (2.19), which, from Royden (1988; page 191), implies $S_{n}$ is compact.

From (2.6) and (2.13), we define the following transformation function:

$h(\boldsymbol{p})=(\boldsymbol{a}, \boldsymbol{b}) \quad$ for $\left\{\begin{array}{l}a_{i j}=p_{i j} / b_{i j} \\ b_{i j}=\sum_{k=i}^{m+1} p_{k j}\end{array} \quad 1 \leq j \leq q, 1 \leq i \leq m_{j}\right.$,

where $\boldsymbol{a}=\left(a_{i j}\right)$ and $\boldsymbol{b}=\left(b_{1 j}\right)$. Note that from $(3.1), h(\boldsymbol{p})$ is well-defined on $\mathcal{E}_{n}$, and that by iteration on $(3.2)$, it can be shown that $h^{-1}$ uniquely exists and is continuous. With some algebraic work, Ren and Riddlesworth (2012) establish the following for $L(\boldsymbol{p})$ in $(2.7)$ :

$$
\begin{aligned}
L(\boldsymbol{p}) & =L\left(h^{-1}(\boldsymbol{a}, \boldsymbol{b})\right) \\
& =\left(\prod_{j=1}^{q}\left(b_{1 j}\right)^{N_{1 j}}\right)\left(\prod_{j=1}^{q} \prod_{i=1}^{m_{j}}\left(a_{i j}\right)^{\delta_{i j}}\left(1-a_{i j}\right)^{N_{i j}-\delta_{i j}}\right) \\
& \equiv G(\boldsymbol{a}, \boldsymbol{b}),
\end{aligned}
$$

which, from (3.1)-(3.2) and (2.8)-(2.9), implies

$$
\begin{aligned}
h\left(\mathcal{E}_{n}\right) & =\left\{h(\boldsymbol{p}) \mid L\left(\boldsymbol{p} \geq c L\left(\hat{F}_{n}\right)\right\}\right. \\
& =\left\{(\boldsymbol{a}, \boldsymbol{b}) \mid(\boldsymbol{a}, \boldsymbol{b}) \in \mathcal{F}_{n}, G(\boldsymbol{a}, \boldsymbol{b}) \geq c L\left(\hat{F}_{n}\right)\right\}
\end{aligned}
$$

where

$$
\begin{aligned}
\mathcal{F}_{n}=\left\{(\boldsymbol{a}, \boldsymbol{b}) \mid 0 \leq a_{i j} \leq 1,0<b_{1 j}<1\right. \\
\left.\quad \text { for } 1 \leq j \leq q, 1 \leq i \leq m_{j} ; \sum_{j=1}^{q} b_{i j}=1\right\} .
\end{aligned}
$$

Since $\log G(\boldsymbol{a}, \boldsymbol{b})$ is concave down, we know that from Bazaraa et al. (1993; page 116), $G(\boldsymbol{a}, \boldsymbol{b})$ is quasiconcave (see definition in Bazaraa et al., 1993; page 108). Thus, if

$$
G\left(\boldsymbol{a}^{(1)}, \boldsymbol{b}^{(1)}\right) \geq c L\left(\hat{F}_{n}\right) \quad \text { and } \quad G\left(\boldsymbol{a}^{(2)}, \boldsymbol{b}^{(2)}\right) \geq c L\left(\hat{F}_{n}\right)
$$

we have that for any $0 \leq \lambda \leq 1$,

$$
\begin{aligned}
& G\left(\lambda\left(\boldsymbol{a}^{(1)}, \boldsymbol{b}^{(1)}\right)+(1-\lambda)\left(\boldsymbol{a}^{(2)}, \boldsymbol{b}^{(2)}\right)\right) \\
& \quad \geq \min \left\{G\left(\boldsymbol{a}^{(1)}, \boldsymbol{b}^{(1)}\right), G\left(\boldsymbol{a}^{(2)}, \boldsymbol{b}^{(2)}\right)\right\} \geq c L\left(\hat{F}_{n}\right) .
\end{aligned}
$$

Hence, $h\left(\mathcal{E}_{n}\right)$ in $(3.4)$ is convex due to $(3.5)$.
From Royden (1988; page 183, Problem 35), we know that the convexity of $h\left(\mathcal{E}_{n}\right)$ implies that $h\left(\mathcal{E}_{n}\right)$ is connected. Thus, the continuity of $h^{-1}$ implies $h^{-1}\left(h\left(\mathcal{E}_{n}\right)\right)=\mathcal{E}_{n}$ is connected; in turn, $S_{n}$ is connected due to continuity of $\tau(\boldsymbol{p})$ (Royden, 1988; page 182). From Royden (1988; page 183), we know that $S_{n}$ is either an interval or a single point. Since $S_{n}$ is compact and since $\tau(\boldsymbol{p})$ is continuous on compact set $\mathcal{E}_{n}$, we know that $S_{n}$ is a closed interval $\left[T_{L}, T_{U}\right]$ with $T_{L}$ and $T_{U}$ given by $(2.20)$.

Proof of Theorem 3. Assume $\theta_{0} \in S_{n}=\left[T_{L}, T_{U}\right]$, where $T_{L}$ and $T_{U}$ are given by (2.20). From the proof of Theorem 2, we know that $\tau(\boldsymbol{p})$ in (2.17) is continuous on $\mathcal{E}_{n}$ in (2.19). Since $T_{L}$ and $T_{U}$ are the lower and upper bound of $\tau(\boldsymbol{p})$ on $\mathcal{E}_{n}$, respectively, we know that from the Intermediate Value Theorem, there exists $\boldsymbol{p}^{*} \in \mathcal{E}_{n}$ such that $\theta_{0}=\tau\left(\boldsymbol{p}^{*}\right)$. From $\boldsymbol{p}^{*} \in \mathcal{E}_{n}$, we know $L\left(\boldsymbol{p}^{*}\right) \geq c L\left(\hat{F}_{n}\right)$, which, from (2.18) and $\theta_{0}=\tau\left(\boldsymbol{p}^{*}\right)$, implies

$$
r\left(\theta_{0}\right)=\left(L\left(\hat{F}_{n}\right)\right)^{-1} \sup _{\boldsymbol{p}}\left\{L(\boldsymbol{p}) \mid \tau(\boldsymbol{p})=\theta_{0}\right\} \geq \frac{L\left(\boldsymbol{p}^{*}\right)}{L\left(\hat{F}_{n}\right)} \geq c .
$$

Assume $r\left(\theta_{0}\right) \geq c$, where $r\left(\theta_{0}\right)$ is given by (2.18). From (2.16)-(2.17), we know that

$$
E_{n}=\left\{\boldsymbol{p} \mid \boldsymbol{p} \text { satisfies }(2.8)-(2.9) \text { and } \tau(\boldsymbol{p})=\theta_{0}\right\}
$$

is not empty. Thus, from (2.18) there exists a sequence of points $\boldsymbol{p}^{(k)} \in E_{n}$ such that

$$
\frac{L\left(\boldsymbol{p}^{(k)}\right)}{L\left(\hat{F}_{n}\right)} \geq r\left(\theta_{0}\right)-\frac{1}{k}
$$

for sufficiently large $k$. Since (3.6)-(3.7) and $r\left(\theta_{0}\right) \geq c$ imply

$$
\tau\left(\boldsymbol{p}^{(k)}\right)=\theta_{0} \quad \text { and } \quad \frac{L\left(\boldsymbol{p}^{(k)}\right)}{L\left(\hat{F}_{n}\right)} \geq c-\frac{1}{k},
$$

and since $\left\{\boldsymbol{p}^{(k)}\right\}$ contains a convergent subsequence, still denoted as $\left\{\boldsymbol{p}^{(k)}\right\}$, such that

$$
\boldsymbol{p}^{(k)} \rightarrow \boldsymbol{p}^{(0)}, \quad \text { as } n \rightarrow \infty
$$

where $\boldsymbol{p}^{(0)}$ satisfies (2.8)-(2.9), we know that the continuity of $L(\boldsymbol{p})$ in $(2.7)$ gives

$$
\frac{L\left(\boldsymbol{p}^{(0)}\right)}{L\left(\hat{F}_{n}\right)}=\lim _{k \rightarrow \infty} \frac{L\left(\boldsymbol{p}^{(k)}\right)}{L\left(\hat{F}_{n}\right)} \geq c>0
$$

Note that from the arguments in (3.1), above (3.10) implies $\sum_{i=1}^{m+1} p_{i \zeta}^{(0)}>0$. Thus, from (2.17) and (3.8)-(3.9) we have

$$
\tau\left(\boldsymbol{p}^{(0)}\right)=\lim _{k \rightarrow \infty} \tau\left(\boldsymbol{p}^{(k)}\right)=\theta_{0}
$$

The proof follows from (2.19) and (3.9)-(3.11). 


\section{ACKNOWLEDGEMENTS}

The authors thank Liang Peng for discussions and providing references during the preparation of this manuscript. The authors also thank the referee for his/her comments on an earlier version of this manuscript.

\section{Received 13 February 2012}

\section{REFERENCES}

BazaraA, M. S., Sherali, H. D. and Shetty, C. M. (1993). Nonlinear Programming, Theory and Algorithms, 2nd ed. John Wiley \& Sons, Inc. MR2218478

Chung, K. L. (1974). A Course in Probability Theory, 2nd ed. Academic Press. MR1796326

Li, G., Li, R. Z. and Zhou, M. (2005). Empirical likelihood in survival analysis. In: Contemporary Multivariate Analysis and Design of Experiments. J. Fan and G. Li, eds. The World Scientific Publisher, pp. 337-350. MR2271092

LI, G. and VAN KeIlegom, I. (2002). Likelihood ratio confidence bands in non-parametric regression with censored data. Scandinavian Journal of Statistics 29 547-562. MR1925574

OWEn, A. B. (1988). Empirical likelihood ratio confidence intervals for a single functional. Biometrika 75 237-249. MR0946049

Owen, A. B. (2001). Empirical Likelihood. Chapman \& Hall/CRC.
REN, J. and Riddlesworth, T. (2012). Bivariate nonparametric maximum likelihood estimator with right censored data. (In submission)

Riddlesworth, T. (2011). Estimation for the Cox Model with Various Types of Censored Data. Ph.D. Dissertation, Department of Mathematics, University of Central Florida.

Royden, H. L. (1988). Real Analysis, 3rd ed. Prentice \& Hall, Inc. MR0928805

Jian-Jian Ren

Department of Mathematics

University of Maryland

College Park

MD 20742

USA

E-mail address: jjren@umd.edu

Tonya Riddlesworth

Department of Mathematics

Tennessee Technological University

110 University Drive

Cookeville, TN 38505

USA 